PROCEEDINGS OF THE

AMERICAN MATHEMATICAL SOCIETY

Volume 127, Number 1, January 1999, Pages $71-78$

S 0002-9939(99)04501-3

\title{
A GENERALIZATION OF THE AUSLANDER-NAGATA PURITY THEOREM
}

\author{
MIRIAM RUTH KANTOROVITZ
}

(Communicated by Wolmer V. Vasconcelos)

\begin{abstract}
Let $B \hookrightarrow A$ be a module finite extension of normal domains. We show that if $B \hookrightarrow A$ is unramified in codimension one and if $A$ has finite projective dimension over $B$, then $A$ is étale over $B$. Our proof makes use of P. Roberts' New Intersection Theorem.
\end{abstract}

\section{INTRODUCTION}

In this paper we give a generalization of the purity of branch locus theorem. The classical purity of branch locus theorem says the following. Let $R$ be a regular local ring and $A$ a normal domain which is a module finite ring extension of $R$. If the extension $R \hookrightarrow A$ is unramified in codimension one, then it is unramified. Following Auslander $[\mathrm{Au}]$, we say that purity holds for a normal domain $R$ if given any finite extension, $R \hookrightarrow S$ with $S$ normal, which is unramified in codimension one, then $S$ is unramified over $R$. Thus, the purity of branch locus theorem states that purity holds for regular local rings. Zariski [Z] gave a proof of this theorem in a geometrical context. A more algebraic proof, based on Chow's local Bertini theorem [Ch], is due to Nagata [N1]. Auslander [Au] gave a different algebraic proof where he reduced the problem to a module theoretic statement. Grothendieck [SGA2] calls a local ring $(R, m)$ pure if the restriction maps of étale covers (i.e. finite unramified flat extensions)

$$
E t(\operatorname{Spec}(A)) \rightarrow E t(\operatorname{Spec}(A) \backslash m)
$$

is an equivalence. Note that this concept of purity is weaker than Auslander's.

Grothendieck proved that complete intersections of dimension at least 3 are pure. Cutkosky $[\mathrm{Cu}]$ showed that a much larger class of rings are pure. He also showed ( $[\mathrm{Cu}]$, Theorem 5 ) that if $R$ is a normal excellent complete intersection and if $R \hookrightarrow A$ is a finite extension which is unramified in codimension two and with $A$ normal, then the extension is unramified (and hence étale, since over normal domains, unramified implies étale).

Griffith [G2] gave examples of Gorenstein rings where purity does not hold, even when the extension is unramified at every non-maximal prime ideal.

Received by the editors October 17, 1996 and, in revised form, May 14, 1997. 1991 Mathematics Subject Classification. Primary 13B15; Secondary $13 B 02$.

Key words and phrases. Auslander-Nagata purity, unramified extension.

(C)1999 American Mathematical Society 
The main result of this paper is:

Theorem 2.1. Let $B \hookrightarrow A$ be a module finite extension of normal domains which is unramified in codimension one, and suppose $A$ has finite projective dimension over $B$. Then $A$ is étale over $B$.

Note that the base ring $B$ is only assumed to be a normal domain. To compensate for easing the restrictions on $B$, we need to assume that $A$ has finite projective dimension over $B$. In the classical case, when $B$ is regular, this hypothesis on $A$ is automatically satisfied.

Our proof uses generalizations of ideas in Auslander's proof and makes use of Roberts' New Intersection Theorem [R].

In the first section of this paper we list definitions and results which are used in the proof of the main theorem. In section 2 we prove the main theorem and give an application related to rigidity of Tor. We also give an example showing that the theorem is sharp in the sense that if we drop the assumption of finite projective dimension, then the extension ring need not even be Cohen-Macaulay (even if the base ring is a complete Gorenstein ring and the extension is unramified on the punctured spectrum).

\section{Preliminaries}

We briefly review some basic definitions and results which will be used in the proof of the main theorem. Standard references are Matsumura [Ma] or Bourbaki [Bou]. Another useful reference is $[\mathrm{BH}]$.

Definition 1.1. Let $M$ be a module over a local ring $(R, m)$. The depth of $M$, denoted by $\operatorname{depth}_{R}(M)$, is defined to be $\inf \left\{i \mid \operatorname{Ext}_{R}^{i}(R / m, M) \neq 0\right\}$.

1.2 (Auslander-Buchsbaum's theorem). Let $M$ be a finitely generated $R$-module of finite projective dimension over $R$. Then

$$
\operatorname{pd}_{R} M+\operatorname{depth}_{R} M=\operatorname{depth}_{R} R
$$

where $\operatorname{pd}_{R} M$ is the projective dimension of $M$ over $R$.

1.3. Let $B \hookrightarrow A$ be a module finite extension of local rings and $M$ a finitely generated A-module.

Then

$$
\operatorname{depth}_{A} M=\text { depth }_{B} M \quad \text { and } \operatorname{dim}_{A} M=\operatorname{dim}_{B} M .
$$

Moreover, $\ell_{B} M<\infty$ if and only if $\ell_{A} M<\infty$.

We say that a property of a ring $R$ holds in codimension $i$ if, for each prime ideal $p$ with $h t p \leq i$, the property holds for the ring $R_{p}$.

A ring $A$ satisfies $R_{i}$ if $A$ is regular in codimension $i$. A finitely generated $A$ module $M$ satisfies $S_{i}$ (or is $S_{i}$ ) if $\operatorname{depth}_{R_{p}}\left(M_{p}\right) \geq \min (i, h t p)$ for all prime ideals $p$ in $R$.

1.4 (Serre's criterion for normal domains). A domain $A$ is normal (i.e. integrally closed in its field of fractions) if and only if $A$ satisfies $R_{1}$ and $S_{2}$.

1.5. Let $A$ be a normal domain and $G$ a subgroup of $A$ ut $(A)$, the group of automorphisms on $A$. Then the ring $A^{G}$ of invariants is a normal domain. 


\subsection{Ramification.}

Definition 1.6. Let $B$ be a ring, $A$ a $B$-algebra, and $P \in \operatorname{Spec}(A) . P$ is said to be unramified over $B$ if $p=P \cap B$ satisfies:

1. $p A_{P}=P A_{P}$

2. $B_{p} / p B_{p} \hookrightarrow A_{P} / P A_{P}$ is a finite separable field extension.

A prime $p \in \operatorname{Spec}(B)$ is unramified in $A$ if every prime $P \in \operatorname{Spec}(A)$ such that $P \cap B=p$ is unramified over $B$. We say $A$ is unramified over $B$ if every ideal $P \in$ $\operatorname{Spec}(A)$ is unramified over $B$, or equivalently, if every $p \in \operatorname{Spec}(B)$ is unramified in $A$. The ring $A$ is étale over $B$ if $A$ is unramified and flat over $B$.

For a more detailed treatment of unramified extensions the reader may wish to consult Auslander-Buchsbaum [AB] or Nagata [N2]. We now recall the following results from $[\mathrm{AB}]$, Section 4.

1.7. Let $B \hookrightarrow A$ be a module finite extension of normal domains. If $A$ is unramified over $B$, then $A$ is flat and hence étale over $B$.

1.8. Let $B$ be a local ring, $B \hookrightarrow A$ be a module finite extension of normal domains which is unramified in codimension one. If $A$ is free as a $B$-module, then $A$ is unramified over $B$.

1.2. Normal extensions and normal closure. Let $B$ be a normal domain and let $B \hookrightarrow A$ be an extension of domains. Denote by $K$ and $L$ the corresponding fraction fields.

Definition 1.9. The extension $B \hookrightarrow A$ is normal if $A$ is normal and the fraction field extension $K \hookrightarrow L$ is Galois.

Let $G$ be the Galois group of $L$ over $K$, referred to as $\operatorname{Gal}(A / B)$. Then $G$ operates on $A$ and the fixed ring $A^{G}$ is $B$.

For $B \hookrightarrow A$ a module finite extension of normal domains (with fraction fields $K$ and $L$ respectively), we construct the normal closure as follows (see Griffith [G1]): Let $E$ be the Galois closure of the field extension $K \hookrightarrow L$ and let $S$ be the integral closure of $A$ in $E$. We call the ring extension $B \hookrightarrow S$ the normal closure of the extension $B \hookrightarrow A$. Note that when $A$ is unramified over $B$ in codimension zero, then $K \hookrightarrow L$ is separable and hence $S$ is a module finite extension of $A$.

The next theorem (see [G2], Section 1) shows that unramification transfers to the normal closure.

Theorem 1.10. Let $B \hookrightarrow A$ be a module finite extension of normal domains. If $B \hookrightarrow A$ is unramified in codimension $i$, then the normal closure $B \hookrightarrow S$ is unramified in codimension $i$ (and so is the extension $A \hookrightarrow S$ ).

1.3. Module structure on $\operatorname{Hom}_{B}(-,-)$ and Module isomorphisms. Let $A$ be a $B$-algebra, $M$ an $A$-module and $N$ a $B$-module. Then $\operatorname{Hom}_{B}(N, M)$ has an $A$ module structure via $M$ as follows: For $a \in A$ and $f \in \operatorname{Hom}_{B}(N, M)$, define $a f$ by $(a f)(n):=a f(n) \in M$ for $n \in N$. This $A$-module structure on $\operatorname{Hom}_{B}(N, M)$ is called codomain induced.

Remark 1.11. When we use the codomain induced module structure, $\operatorname{Hom}_{B}(N,-)$ becomes a functor from the category of $A$-modules to itself. 
In $[\mathrm{Au}]$, Auslander gives a module theoretic proof of the classical purity of branch locus theorem of Nagata [N1]. He first reduces the problem to the case of a normal extension. Then he uses the following module isomorphism:

Lemma 1.12. Let $B \hookrightarrow S$ be a normal extension of normal domains with Galois group $G=\operatorname{Gal}(S / B)$ of order $g=|G|$. Then $\operatorname{Hom}_{B}(S, S) \simeq_{S} S^{g}$, where $S^{g}$ is a free $S$-module of rank $g$ and by $\simeq_{S}$ we mean isomorphism as $S$-modules (the $S$-module structure on $\operatorname{Hom}_{B}(S, S)$ is codomain induced).

In our situation, $B \hookrightarrow A$ is a module finite extension of normal domains which is not necessarily a normal extension. By passing to the normal closure $B \hookrightarrow A \hookrightarrow S$ and using the above isomorphism, Borek [Bor] obtained additional useful module isomorphisms.

Theorem 1.13 (Borek [Bor], Section 3). Let $B$ be a local normal domain. Let $B \hookrightarrow A$ be a module finite extension of normal domains which is unramified in codimension one, $B \hookrightarrow S$ the normal closure, and $W$ a finitely generated $S$-module which is $S_{2}$. Then

$$
\operatorname{Hom}_{B}(A, W)^{h} \simeq_{S} \operatorname{Hom}_{A}\left(S, \operatorname{Hom}_{B}(A, W)\right),
$$

where the $S$-module structure on $\operatorname{Hom}(-,-)$ is codomain induced and $h$ is the order of the Galois group $\operatorname{Gal}(S / A)$.

Remark 1.14. Using the adjoint isomorphism we have:

$$
\begin{aligned}
\operatorname{Hom}_{B}(A, W)^{h} & \simeq_{S} \operatorname{Hom}_{A}\left(S, \operatorname{Hom}_{B}(A, W)\right) \\
& \simeq_{S} \operatorname{Hom}_{B}\left(S \otimes_{A} A, W\right) \simeq_{S} \operatorname{Hom}_{B}(S, W),
\end{aligned}
$$

where the last isomorphism is an $S$-module isomorphism by Remark 1.11.

Remark 1.15. When we take $W$ to be $S$ (which is a normal domain and a module finite extension of $B$ and hence $S_{2}$ over $B$ by Theorem 1.4) we get from Remark 1.14:

$$
\operatorname{Hom}_{B}(A, S)^{h} \simeq_{S} \operatorname{Hom}_{B}(S, S) \simeq_{S} S^{g} .
$$

Hence $\operatorname{Hom}_{B}(A, S)$ is projective as an $S$-module.

1.4. New Intersection Theorem. A key ingredient in the proof of our main theorem is Roberts' New Intersection Theorem.

Theorem 1.16 (Roberts $[\mathrm{R}])$. Let $R$ be a local ring with maximal ideal $m$. Suppose

$$
F_{*}: \quad 0 \rightarrow F_{s} \stackrel{\phi_{s}}{\longrightarrow} F_{s-1} \stackrel{\phi_{s-1}}{\longrightarrow} \ldots \rightarrow F_{1} \stackrel{\phi_{1}}{\longrightarrow} F_{0} \rightarrow 0
$$

is a complex of finitely generated free $R$-modules such that

1. $\phi_{i}: F_{i} \rightarrow F_{i-1}$ is represented by a matrix with entries in the maximal ideal $m$ for all $i$.

2. The length of the homology modules, $\ell_{R}\left(H_{i}\left(F_{*}\right)\right)$, is finite for all $i$.

If $s<\operatorname{dim} R$, then $F_{*}$ is exact.

Note. $F_{*}$ exact means $F_{i}=0$ for $0 \leq i \leq s$. To see this, consider first $F_{1} \stackrel{\phi_{1}}{\rightarrow} F_{0} \rightarrow 0$. Since $F_{0}$ is free, this exact sequence splits, so $\phi_{1}$ has a right inverse $\psi_{1}, \phi_{1} \circ \psi_{1}=$ $i d_{F_{0}}$. But, unless $F_{0}=0$, this is impossible since the entries of $\phi_{1}$ are in $m$. Hence $F_{0}=0$, and by induction we have $F_{0}=F_{1}=\cdots=F_{s}=0$. 


\section{MAIN THEOREM}

Theorem 2.1. Let $B \hookrightarrow A$ be a module finite extension of normal domains which is unramified in codimension one, and suppose $\operatorname{Pd}_{B} A<\infty$. Then $A$ is étale over $B$.

Proof. Since $B$ is a normal domain, it is enough to show that $A$ is unramified over $B(1.7)$. Let $p$ be a prime in $B$. We show $p$ is unramified in $A$ by induction on the height of $p$.

When $h t p \leq 1$, the assertion is true by assumption.

Suppose $h t p \geq 2$. Localizing at $p$ we get that $B_{p} \hookrightarrow A_{p}$ is a module finite extension of normal domains which is unramified in codimension one and $P d_{B_{p}} A_{p}$ $<\infty$. Since $p$ is unramified in $A$ if and only if $A_{p}$ is unramified over $B_{p}$, we may assume that $B$ is local, $p$ the maximal ideal of $B, \operatorname{dim} B \geq 2$ and $\operatorname{Pd}_{B} A<\infty$. For this situation, it is enough to show (by 1.8) that $A$ is a free $B$-module.

First we take the normal closure of $B \hookrightarrow A$ :

$$
B \hookrightarrow A \hookrightarrow S
$$

Let $G$ be the Galois group $\operatorname{Gal}(S / B)$ and let $H$ be $\operatorname{Gal}(S / A)$. We denote the orders of $G$ and $H$ by $g$ and $h$ respectively. Then $B \hookrightarrow S$ is a module finite (normal) extension of normal domains which is unramified in codimension one (Theorem 1.10). (Note that we do not have $P d_{B} S<\infty$ anymore, but we gained the Galois group action.) Let $q \in \operatorname{Spec} B \backslash\{p\}$, then by induction, $q$ is unramified in $A$, so $A_{q}=A \otimes_{B} B_{q}$ is a free $B_{q}$-module. Therefore, for any finitely generated $B$ module $M$, we have:

$$
\operatorname{Ext}_{B}^{i}(A, M)_{q}=\operatorname{Ext}_{B_{q}}^{i}\left(A_{q}, M_{q}\right)=0 \text { for all } i>0 .
$$

In particular, when $M$ is taken to be $S, \operatorname{Ext}_{B}^{i}(A, S)_{q}=0$ for every $q \in \operatorname{Spec} B \backslash\{p\}$. Hence $\ell_{B}\left(\operatorname{Ext}_{B}^{i}(A, S)\right)<\infty$ for every $i>0$.

We now consider the $B$-modules $\operatorname{Ext}_{B}^{i}(A, S)$ for $i>0$. Let

$$
F_{*}: 0 \rightarrow F_{d} \stackrel{\phi_{d}}{\longrightarrow} F_{d-1} \stackrel{\phi_{d-1}}{\longrightarrow} \ldots \rightarrow F_{1} \stackrel{\phi_{1}}{\rightarrow} F_{0} \stackrel{\epsilon}{\rightarrow} A \rightarrow 0
$$

be a minimal free resolution of $A$ over $B$ (where $d=P d_{B} A$ ). Then for all $i, \phi_{i}$ may be represented by a matrix with entries in $p$, the maximal ideal of $B$. We apply $\operatorname{Hom}_{B}(-, S)$ to $F_{*}$ and get:

$$
\begin{aligned}
L_{*}: 0 & \rightarrow \operatorname{Hom}_{B}(A, S) \stackrel{\epsilon^{*}}{\rightarrow} \operatorname{Hom}_{B}\left(F_{0}, S\right) \\
& \stackrel{\phi_{1}^{*}}{\rightarrow} \operatorname{Hom}_{B}\left(F_{1}, S\right) \rightarrow \ldots \stackrel{\phi_{d}^{*}}{\rightarrow} \operatorname{Hom}_{B}\left(F_{d}, S\right) \rightarrow 0
\end{aligned}
$$

with $L_{i}=\operatorname{Hom}_{B}\left(F_{i}, S\right)$ for $i=0, \ldots, d$ and $L_{-1}=\operatorname{Hom}_{B}(A, S)$. Note:

1. $L_{i} \simeq_{S} S^{r_{i}}$ where $r_{i}$ is the rank of the free $B$-module $F_{i}$ for $i=0, \ldots, d$ and by Remark $1.15, L_{-1}^{h} \simeq{ }_{S} S^{g}$.

2. The induced $B$-module maps $\phi_{i}^{*}$ and $\epsilon^{*}$ are also $S$-module maps by Remark 1.11 .

Let $m \in$ Spec $S$ be a maximal ideal lying over $p$. Localizing the complex $L_{*}$ at $m$ yields:

$$
\left(L_{*}\right)_{m}: 0 \rightarrow\left(L_{-1}\right)_{m} \stackrel{\epsilon^{*}}{\rightarrow}\left(L_{0}\right)_{m} \stackrel{\phi_{1}^{*}}{\rightarrow}\left(L_{1}\right)_{m} \rightarrow \ldots \stackrel{\phi_{d}^{*}}{\rightarrow}\left(L_{d}\right)_{m} \rightarrow 0
$$

This is a complex of finitely generated free $S_{m}$-modules such that $H^{i}\left(\left(L_{*}\right)_{m}\right)=$ $\operatorname{Ext}_{B}^{i}(A, S)_{m}$ (which is a finite length $B$-module by the above discussion) for $i \geq 0$. 
Our goal is to apply the new intersection theorem to a modified version of the complex $\left(L_{*}\right)_{m}$. Since $\phi_{i}^{*}$ is just multiplication by the transpose matrix of $\phi_{i}$, we have that $\phi_{i}^{*}$ has entries in $p S_{m} \subset m S_{m}$ for $i=1, \ldots, d$. But $\epsilon^{*}$ may not have all its entries in $m S_{m}$. To fix that, let $C=\operatorname{Coker}\left(\epsilon^{*}\right)$. Since $\operatorname{Hom}(-, S)$ is left exact,

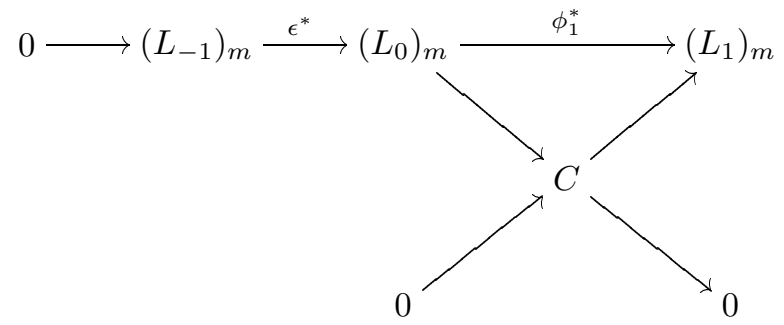

is exact and $P d_{S_{m}} C \leq 1$.

Let

$$
0 \rightarrow\left(\tilde{L}_{-1}\right)_{m} \stackrel{\phi_{0}^{*}}{\rightarrow}\left(\tilde{L}_{0}\right)_{m} \rightarrow C \rightarrow 0
$$

be a minimal free resolution of $C$ over $S_{m}$ (where we allow $\tilde{L}_{-1}$ to be 0 if $P d_{S_{m}} C$ $=0)$. So $\phi_{0}^{*}$ has entries in $m S_{m}$. Hence, in $\left(L_{*}\right)_{m}$, we may replace the exact sequence $0 \rightarrow\left(L_{-1}\right)_{m} \stackrel{\epsilon^{*}}{\rightarrow}\left(L_{0}\right)_{m}$ by $0 \rightarrow\left(\tilde{L}_{-1}\right)_{m} \stackrel{\phi_{0}^{*}}{\rightarrow}\left(\tilde{L}_{0}\right)_{m}$ without changing the homology. We denote the new complex again by $\tilde{L}_{* m}$, which is now a complex of finitely generated free $S_{m}$-modules with all maps having entries in the maximal ideal, and with homologies:

$$
H^{i}\left(\left(\tilde{L_{*}}\right)_{m}\right)= \begin{cases}0 & \text { for } i=-1,0 \\ \left(\operatorname{Ext}_{B}^{i}(A, S)\right)_{m} & \text { for } i=1, \ldots, d .\end{cases}
$$

Next we check that $\left(\tilde{L_{*}}\right)_{m}$ satisfies the hypothesis of the new intersection theorem.

Since the length of $\operatorname{Ext}_{B}^{i}(A, S)$ over $B$ is finite for every $i>0$ and $B \hookrightarrow S$ is a module finite extension, we have that the length of $\operatorname{Ext}_{B}^{i}(A, S)$ over $S$ is also finite (1.3). Hence, when localizing, we still have $\ell_{S_{m}}\left(\operatorname{Ext}_{B}^{i}(A, S)_{m}\right)<\infty$ for $i=1, \ldots, d$. So in any case, $\ell_{S_{m}}\left(H^{i}\left(\left(\tilde{L_{*}}\right)_{m}\right)\right)<\infty$ for all $i$.

Finally, the length of the complex $\left(\tilde{L_{*}}\right)_{m}$ is $d+1$, where $d=\operatorname{Pd}_{B} A$. By Auslander-Buchsbaum's theorem (1.2) we have:

$$
P d_{B} A+\operatorname{depth}_{B} A=\operatorname{depth} B \leq \operatorname{dim} B=\operatorname{dim} S_{m}
$$

where the last equality holds since $S$ is module-finite over $B$.

Since $A$ is a normal domain, it satisfies $S_{2}$ and hence $\operatorname{depth}_{B} A \geq 2$ (here we use $\operatorname{dim} B \geq 2)$. So the length of $\left(\tilde{L_{*}}\right)_{m}$ is $d+1<P d_{B} A+\operatorname{depth}_{B} A \leq \operatorname{dim} S_{m}$, by (*). Now we can apply the New Intersection Theorem to $\left({\tilde{L_{*}}}_{{ }}{ }_{m}\right.$ to get:

$$
0=\left(\tilde{L_{1}}\right)_{m}=\left(\operatorname{Hom}_{B}\left(F_{1}, S\right)\right)_{m} \simeq S_{m}^{r_{1}} \quad \text { where } r_{1}=\operatorname{rank} F_{1} .
$$

Therefore $F_{1}=0$, and the minimal free resolution $F_{*}$ of $A$ over $B$ is just $0 \rightarrow F_{0} \rightarrow$ $A \rightarrow 0$. So $A$ is free over $B$.

We now give an easy application of a theorem by Huneke-Wiegand on rigidity of Tor [HW2, Theorem 2.7] (see also [HW1] and [Mi]). The theorem says the following:

Theorem 2.2 (Huneke-Wiegand). Let $R$ be a hypersurface, and let $M$ and $N$ be two $R$-modules. If $M \otimes_{R} N$ is reflexive, then $M$ and $N$ are reflexive, $\operatorname{Tor}_{i}^{R}(M, N)=$ 0 for all $i>0$ and either $M$ or $N$ has finite projective dimension. 
Application 2.3. Let $B$ be a hypersurface which is a normal domain. Let $B \hookrightarrow A$ be a module finite extension of normal domains that is unramified in codimension one. If $A \otimes_{B} A$ is reflexive, then $A$ is étale over $B$.

Proof. By Theorem 2.2, $A \otimes_{B} A$ reflexive implies $P d_{B} A<\infty$ and hence, by Theorem 2.1, $A$ is étale over $B$.

In the following example we show that Theorem 2.1 is sharp in the sense that it is false if we drop the condition of finite projective dimension, even if the base ring is a Gorenstein complete local normal domain.

Example 2.4 (see Fossum [F], Example 16.5). Let $A=k\left[X_{1}, \ldots, X_{d}\right]$ be the polynomial ring in $d$ variables (with $d \geq 2$ ) over a field $k$ which contains a primitive $n^{\text {th }}$ root of unity, $\omega$, such that $n$ is relatively prime to the characteristic of $k$. Let $\sigma$ be the automorphism on $A$ defined by $\sigma\left(X_{i}\right)=\omega X_{i}$ and let $G=\langle\sigma\rangle$ be the cyclic group generated by $\sigma$. Then $A^{G}=B$, the ring of invariants under $G$, is generated as a $k$-algebra by the monomials of degree $n$, and if $n=d$, then $B$ is Gorenstein (see Watanabe $[\mathrm{W}]$ ). The extension $B \hookrightarrow A$ is unramified on the punctured spectrum of $B$, i.e. at every non-maximal prime ideal (see [Bor], Section 5), but its not unramified since $A$ is regular and $B$ is not.

\section{REFERENCES}

[Ab] S. Abhyankar, in Ramification Theoretic Methods in Algebraic Geometry, Annals of Mathematics Studies Vol. 43, Princeton: Princeton Univ. Press, 1959. MR 21:4158

[Au] M. Auslander, On the purity of branch locus, Amer. J. Math. 84 (1962), 116-125. MR 25:1182

[AB] M. Auslander and D. Buchsbaum, On ramification theory in Noetherian rings, Amer. J. Math. 81 (1959), 749-765. MR 21:5659

[Bor] A. Borek, Weak purity for Gorenstein rings, J. of Alg. 175 (1995), 409-450. MR 96i: 13010

[Bou] N. Bourbaki, Commutative Algebra, Chapters 1-7, Berlin, Heidelberg, New York: Springer-Verlag, 1989. MR 90a:13001

[BH] W. Bruns and J. Herzog, Cohen Macaulay Rings, Cambridge: Cambridge University Press, 1993. MR 95h:13020

[Ch] W. L. Chow, On the theorem of Bertini for local domains, Proceedings of the National Academy of Science, USA, 44 (1958), 580-584. MR 20:3150

[Cu] S. D. Cutkosky, Purity of the branch locus and Lefschetz theorems, Compositio Math. 96, No 2 (1995), 173-195. MR 96h:13023

[F] R. Fossum, The Divisor Class Group of Krull Domains, Ergebnisse der Mathematik und ihrer Grenzgebiete, Vol 74, Berlin, Heidelberg, New York: Springer-Verlag, 1973. MR 52:3139

[G1] P. Griffith, Normal extensions of regular local rings, J. of Alg. 106 (1987), 465-475. MR 88c: 13020

[G2] P. Griffith, Some results in local rings in ramification in low codimension, J. of Alg. 137 (1991), 473-490. MR 92c:13017

[SGA2] A. Grothendieck, Cohomologie locale des faisceaux cohérents et théorèmes de Lefschetz locaux et globaux in Séminaire de Géometrie Algébrique (SGA), fasc. I and fasc. II, IHES, 1962, 1963. MR 57:16294; MR 35:1604

[HW1] C. Huneke and R. Wiegand, Tensor products of modules and the rigidity of Tor, Math. Ann. 299 (1994), 449-476. MR 95m:13008

[HW2] C. Huneke and R. Wiegand, Tensor products of modules, rigidity, and local cohomology, submitted.

[Ma] H. Matsumura, Commutative Ring Theory, Cambridge: Cambridge University Press, 1989. MR 90i: 13001

[Mi] C. Miller, Hypersurface sections: A study of divisor class groups and the complexity of tensor products, Ph. D. thesis, University of Illinois at Urbana-Champaign, 1996. 
[N1] M. Nagata, On the purity of branch locus in regular local rings, Ill. Jour. of Math. 3 (1959), 328-333. MR 21:5660

[N2] M. Nagata, Local Rings, Interscience Tracts in Pure and Applied Mathematics, Vol. 13, New York: Wiley, 1962. MR 27:5790

[R] P. Roberts, Le théorème d'intersection, C. R. Acad. Sc. Paris Sér. I 304 (1987), 177180. MR 89b: 14008

[W] K. Watanabe, Certain invariant subrings are Gorenstein I, Osaka J. Math. 11 (1974), 1-8. MR 50:7124

[Z] O. Zariski, On the purity of branch locus of algebraic functions, Proc. Nat. Acad. U.S.A. 44 (1958), 791-796. MR 20:2344

Department of Mathematics, University of Illinois at Urbana-Champaign, Urbana, ILLINOIS 61801

E-mail address: ruth@math.uiuc.edu 